\title{
Managing pain and fear: Playing your CARDs to improve the vaccination experience
}

\author{
Anna Taddio ${ }^{1 *}$, Anthony llersich², C Meghan McMurtry ${ }^{3}$, Lucie M Bucci ${ }^{4}$, Noni E MacDonald ${ }^{5}$
}

\begin{abstract}
Most vaccinations are administered with a needle, which can cause pain and pain-related symptoms such as fear and fainting. At present, interventions aimed at preventing pain and associated symptoms are not systematically integrated in the vaccination delivery process even though they contribute to negative experiences with vaccination and vaccination noncompliance. In this article, a novel framework for vaccination delivery called the CARD ${ }^{\mathrm{TM}}$ system was reviewed. CARD is an acronym for Comfort, Ask, Relax and Distract, whereby each letter category incorporates evidence-based interventions to reduce pain and fear and related symptoms. CARD can be integrated in usual vaccination planning and delivery activities in many settings to improve the vaccination experience and decrease pain and fear as barriers to vaccination. Immunizers in all settings and organizational leaders are invited to review their vaccination services against CARD to identify opportunities for enhancing the quality of care being provided.
\end{abstract}

Suggested citation: Taddio A, llersich A, McMurtry CM, Bucci LM, MacDonald NE. Managing pain and fear: Playing your CARDs to improve the vaccination experience. Can Commun Dis Rep 2021;47(1):87-91.

https://doi.org/10.14745/ccdr.v47i01a12

Keywords: vaccination, pain, fear, pain management, vaccine hesitancy, needlesticks

\section{Introduction}

Vaccine injections are frequently associated with pain and pain-related adverse effects, such as fear, fainting, nausea and other stress-related responses $(1,2)$. Until recently, little attention has been paid to reducing pain and related symptoms during vaccination. However, accumulating evidence shows that negative experiences with vaccination can contribute to the development of needle fears, vaccine hesitancy and healthcare avoidance behaviours, including vaccination noncompliance $(3,4)$. This is particularly important during childhood, when concerns about pain and fear of needles are high and attitudes towards healthcare providers are being shaped $(2,5)$.

Vaccination is the most common reason, by far, why people receive needles. The World Health Organization has identified overcoming barriers to immunizations as a priority for global health (6). Addressing barriers to vaccination is even more relevant now, during the pandemic, to help with acceptance of COVID-19 vaccine(s) when they become available.

There are numerous negative consequences of unmanaged pain when receiving needles. Individuals are often subjected to longer procedure times and increased use of restraint, and can experience potentially serious adverse events such as fainting, nausea and other stress-related responses $(2,7,8)$. Having to deal
This work is licensed under a Creative Commons Attribution 4.0 International License.

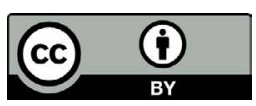

Affiliations

${ }^{1}$ Leslie Dan Faculty of Pharmacy, University of Toronto, Toronto, ON

2 Faculty of Math, University of Waterloo, Waterloo, ON

${ }^{3}$ Department of Psychology, University of Guelph, Guelph, ON

${ }^{4}$ Immunize Canada, Canadian Public Health Association, Ottawa, ON

${ }^{5}$ Faculty of Medicine, Dalhousie University, Halifax, NS

\section{${ }^{\star}$ Correspondence:}

anna.taddio@utoronto.ca with long and complex patient interactions leads to additional stress for the healthcare providers administering vaccinations. Immunizers commonly report challenges with current vaccination delivery processes that may increase the risk of unwanted outcomes. These challenges include suboptimal physical spaces, lack of preparation and communication of important stakeholders, unclear roles, competing demands and excessive patient symptoms (fear, pain, dizziness), particularly in children (9-11). Recently, fear of acquiring COVID-19 infection while being immunized has only exacerbated these concerns.

There are numerous evidence-based and feasible interventions for improving the vaccination experience (1). Pain management needs to be recognized as a part of good vaccination practice and this knowledge needs to be systematically integrated into practice (12). Based on their clinical practice guideline (1), the national HELPinKids\&Adults team recently developed a vaccine delivery framework called CARD ${ }^{\mathrm{TM}}$ that shows immunizers and program managers responsible for vaccination delivery how to integrate these interventions into vaccination planning and delivery processes. In addition, CARD teaches patients how to cope with their own vaccination experience (11). This article explains the framework and how to apply it in various settings. 


\section{What is CARD?}

CARD stands for Comfort, Ask, Relax and Distract. Each of the four initial letters stands for an intervention category, and the four encompass activities that reduce pain, fear, fainting and related symptoms before, during and after vaccination. The CARD system can be used with children and adults, with participatory activities in all four intervention categories.

\section{How was CARD developed?}

CARD was originally designed to improve the vaccination experience at school, but it is a valuable tool for vaccination delivery in various settings, including healthcare providers' offices. In fact, one of its main strengths lies in its adaptability to many different settings. Most importantly, CARD was developed with input from different stakeholders involved in vaccinations at school. Immunizers, students, parents/guardians and school staff were involved in a stepwise approach that included identifying needs and preferences, developing tools and resources, and implementing and evaluating the impact of implementation (11).

\section{CARD works}

In a controlled cluster trial conducted in Niagara, Ontario, students in schools where CARD was implemented (versus control) reported less fear (odds ratio $[O R]=0.47,95 \%$ confidence interval [Cl]: 0.27-0.82) and dizziness (OR=0.26, 95\% $\mathrm{Cl}$ : 0.07-0.91) during vaccination (13). Students educated about CARD had higher knowledge scores and more positive attitudes towards vaccination. Students wanted other students to learn about CARD as they had found it so helpful (13). Immunizers, parents/guardians and school staff also reported more positive attitudes about the vaccination experience when CARD was in use (14).

\section{Tailoring CARD to your setting}

CARD can be tailored to work in a variety of settings, including private offices, hospitals, schools and pharmacies. Key elements of CARD include education of immunizers and patients, setting up the vaccination site to be supportive, and ensuring that immunizer and patient interactions embrace the patients' preferences (i.e. CARD choices). Patients can learn about CARD from online resources, for example, videos and pamphlets (11). While the majority of resources are primarily focused on adolescents, new resources are currently being developed for the adult vaccination context (https://immunize.ca/card-adults). Figure 1 shows sample interventions that patients can "play" to make the procedure a more positive experience.

Immunizers also receive simple training in the importance of the components of CARD and how to support the choices patients make. The immunizer and patient form a team to make the experience as positive as possible. From the planning stages to actual injection, and across different vaccination settings, the immunizer can review current vaccination procedures against the CARD framework, looking for opportunities to incorporate ways to optimize the vaccination experience.

Table 1 summarizes some of the activities normally associated with planning and delivery of vaccinations and how to incorporate CARD into those activities. All stakeholders involved in the vaccination process, including immunizers and patients, can "play their CARDs" to facilitate a more positive vaccination experience. For instance, immunizers can make sure there is comfortable seating for the patient (Comfort) and invite patients to ask questions before, during and after the procedure (Ask). Doing this helps patients feel comfortable, informed and involved, which helps them feel calm. It also builds trust in healthcare providers because they demonstrate that they are caring and attentive to patient needs. Immunizers can also ask patients questions (e.g. How afraid are you? Do you prefer to look away?) to help them assess the patient's status as well as to engage patients as active participants. Patients can bring a favourite item (Comfort) or an electronic device (Distract) for use

Figure 1: Sample interventions from the CARD system

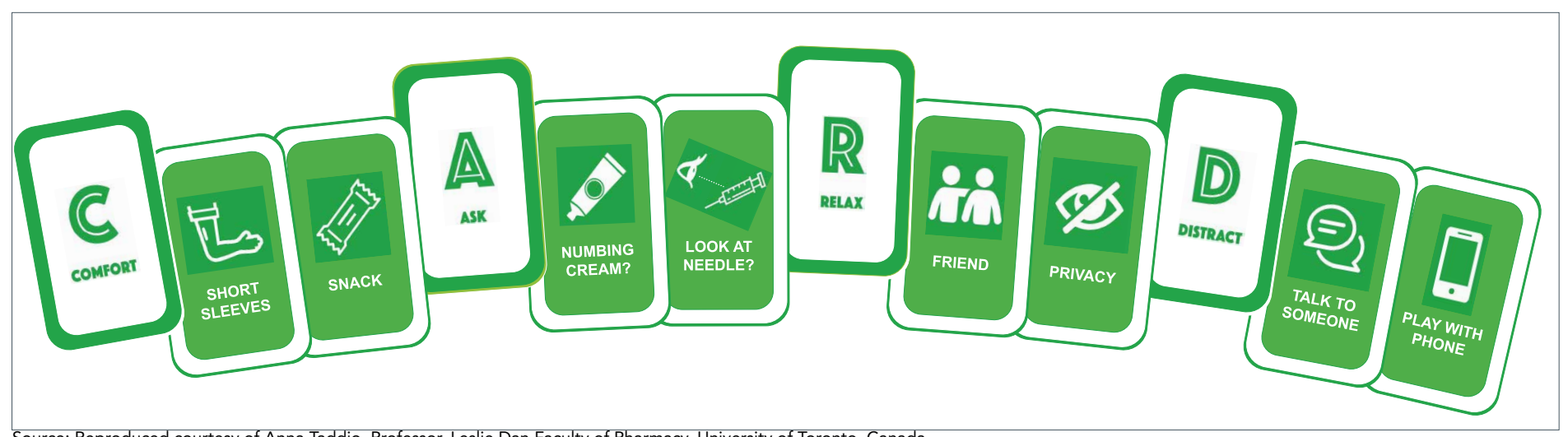




\section{Table 1: CARD system framework for vaccination delivery}

$\begin{gathered}\text { Phase of } \\ \text { vaccination }\end{gathered}$
Preparation/planning

- Esthetic room, free of hazards

- Temperature control

- Accommodates equipment and supplies

Ensure adequate clinic space down

- Allows for privacy

- Allows for no interruptions

- Allows for ability to accommodate a support person with seating

\section{Educate}

patients

and other

stakeholders

(e.g. parents/

guardians,

teachers)

- CARD education (e.g. discussion, tools)

- Answer patient questions

- Book vaccination appointment

\begin{tabular}{l|l} 
Vaccination day & $\begin{array}{l}\text { Patients ask questions they have about vaccination } \\
\text { or coping interventions }\end{array}$ \\
reminders & $\begin{array}{l}\text { Patients plan how they will play their selected } \\
\text { coping strategies (e.g. bring cell phone to use as a } \\
\text { distraction item, wear short-sleeved shirt to allow } \\
\text { for easy access to arm and to increase comfort) }\end{array}$
\end{tabular}

Vaccination day

- Use separate areas for waiting, vaccination and post-vaccination with chairs

- Allow for privacy (e.g. use window coverings, physical barriers)

- Ensure safety measures are in place to prevent transmission of infectious diseases (e.g. sanitization items, face coverings)

Vaccination clinic set-up

- Make sure patients have comfortable seating and are able to be in a reclining position

- Allow patients to use distraction aids or comfort items

- Allow patients to bring a support person

- Arrange seating at clinic tables so that patients do not face each other or equipment, and obscure frightening equipment from site (e.g. use towel, table-top poster)

- Foster a calm environment and be positive

- Review patients' medical history, including fainting and level of fear or worry about vaccination

- Answer patients' questions

- Communicate using neutral language. Do not use words that elicit fear (e.g. the needle "stings") and do not use repetitive reassurance (i.e. don't worry, it's ok, you'll be fine)

- Provide balanced information. Do not suggest that vaccination will not hurt; instead, describe

Vaccination administration sensations (e.g. "pressure" and "pinch") and duration (e.g. "about 1 second") and invite patients to report on how they feel

- Ask patients about their preferences. Do not impose coping interventions such as verbal distraction, taking deep breaths, looking away during injection (these interventions are counter to preferred coping strategies of many individuals and lead to increased levels of fear or distress)

- Ask patients what CARDs they are playing and accommodate requests (e.g. topical anesthetic, support person, private room, injection of two vaccines in same arm)

\section{Table 1: CARD system framework for vaccination delivery (continued)}

Phase of

vaccination

Immunizer activity

Vaccination day (continued)

- Provide distraction agents for patients that do not have them but would like to be distracted (in keeping with infection control and prevention guidelines)

- Ask patients about their preference with respect to the arm to vaccinate. If there is no preference, inject the non-dominant arm

- Ask patients about their preference with respect to injecting two vaccines in the same arm

- Encourage patients to relax their arm so that it is loose and jiggly

- Consider not using alcohol to cleanse the skin as this step is unnecessary, adds time and can increase anticipatory stress ${ }^{b}$

Vaccination administration (continued)

- Inject patients sitting upright (on a parent's/ guardian's lap if patient is a young child)

- Inject vaccines quickly, without aspiration

- If there are multiple injections, administer the most painful vaccine last

- Monitor patient symptoms after vaccination. Suggest muscle tension to patients who are dizzy or prone to fainting (this can be achieved by squeezing legs together or lying down in a reclining chair or on a gym mat)

- Counsel patient regarding post-injection reactions and use of acetaminophen

- Document symptoms and feedback to inform future vaccination ${ }^{c}$

a Resources/tools available online (11)

b See World Health Organization. WHO best practices for injections and related procedures toolkit. Geneva (Switzerland): WHO Document Production Services; 2010 (15)

"See Appendix 5, page 2 of Taddio et al. (1)

Source: Reproduced courtesy of Anna Taddio, Professor, Leslie Dan Faculty of Pharmacy, University of Toronto, Canada

during vaccination to help cope. While each stakeholder (needle givers and receivers) has their own CARDs to play, the full potential of the CARD framework is realized when all play their own CARDs with the shared goal of improving the vaccination experience.

\section{Playing CARD is teamwork}

In many settings, vaccination delivery is complex, and immunizers are unable to make the necessary changes to adopt CARD on their own. It is crucial to involve organizational leaders who can facilitate adoption by making changes to relevant policies and procedures. These changes could include staff roles, training, ongoing communication and evaluation (e.g. staff meetings, summaries, audit and feedback) and ongoing support (e.g. educational resources) (11). This also includes promoting awareness and understanding of overarching models of healthcare delivery and professional standards that promote person-centred care and evidence-based practice, and actively practising continuous quality improvement and reflective practice.

Immunizers and their organizational leaders can also identify opportunities to leverage current activities to facilitate activities 
specific to CARD. For instance, immunizers typically notify teachers and parents/guardians of upcoming school-based vaccinations. These stakeholders can learn about CARD and reinforce teaching the CARD system to the children. Engaging stakeholders, including teachers and parents/guardians, has multiple benefits, including improving fidelity of implementation, creating a "social norm" that recognizes and respects individuals' participation in their healthcare, their preferences for information and coping, and it minimizes the need for additional resources. In turn, parents/guardians and teachers feel more at ease knowing that children are being cared for, and this creates a more welcoming environment for everyone.

Our experience with using CARD in a school-based vaccination program in Niagara, Ontario, was that, after training and support during initial implementation, the system could be incorporated into usual activities in a cost-neutral manner (12). Immunizers will need some additional time to prepare for vaccinations because of the planning steps, such as educating all stakeholders. However, the required time will lessen as everyone becomes familiar with CARD.

\section{Conclusion}

Addressing pain and associated stress-related reactions are proven to improve the vaccination experience for patients and immunizers alike. The long-term benefits of the CARD framework are numerous and include the potential for improved health outcomes due to improved acceptance of healthcare interventions, including vaccination. CARD allows immunizers to "play their best hand" with respect to setting up and running clinics or individual vaccination appointments. The CARD system is a valuable tool for optimizing the vaccination experience and addressing one of the long-recognized yet neglected harms of vaccination, the needle.

\section{Authors' statement}

AT - Conceptualization, writing-original draft, review and editing

Al - Conceptualization, writing-review and editing

$\mathrm{CMM}$ - Conceptualization, writing-review and editing

LMB - Writing-review and editing

NM - Writing-review and editing

All authors take responsibility for the content of this article.

\section{Competing interests}

AT reports a Section 9 Trademark No. 924835 for CARD. LMB reports that Immunize Canada received grants from Pfizer Canada, Merck Canada, GSK Canada, Seqirus Canada and Sanofi Pasteur outside the submitted work. There are no other disclosures.

\section{Acknowledgements}

The authors thank $\mathrm{M}$ Ho for creation of Figure 1.

\section{Funding}

This work is funded by the Canadian Institutes of Health Research (FRN 159905).

The content and view expressed in this article are those of the authors and do not necessarily reflect those of the Government of Canada.

\section{References}

1. Taddio A, McMurtry CM, Shah V, Riddell RP, Chambers CT, Noel M, MacDonald NE, Rogers J, Bucci LM, Mousmanis $P$, Lang E, Halperin SA, Bowles S, Halpert C, Ipp M, Asmundson GJ, Rieder MJ, Robson K, Uleryk E, Antony MM, Dubey V, Hanrahan A, Lockett D, Scott J, Bleeker EV; HELPinKids\&Adults. Reducing pain during vaccine injections: clinical practice guideline. CMAJ 2015;187(13):975-82. DOI PubMed

2. McMurtry CM, Pillai Riddell R, Taddio A, Racine N, Asmundson GJ, Noel M, Chambers CT, Shah V; HELPinKids\&Adults Team. HELPinKids\&Adults Team. Far from "just a poke": common painful needle procedures and the development of needle fear. Clin J Pain 2015;31(10 Suppl):S3-11. DOI PubMed

3. Taddio A, Ipp M, Thivakaran S, Jamal A, Parikh C, Smart S, Sovran J, Stephens D, Katz J. Survey of the prevalence of immunization non-compliance due to needle fears in children and adults. Vaccine 2012;30(32):4807-12. DOI PubMed

4. MacDonald NE; SAGE Working Group on Vaccine Hesitancy. Vaccine hesitancy: Definition, scope and determinants. Vaccine 2015;33(34):4161-4. DOI PubMed

5. Taddio A, llersich AF, llersich AN, Wells J. From the mouth of babes: getting vaccinated doesn't have to hurt. Can J Infect Dis Med Microbiol 2014;25(4):196-200. DOI PubMed

6. World Health Organization. Ten threats to global health in 2019. Geneva: WHO; 2019 (accessed 2020-12-15). https://www.who.int/news-room/spotlight/ten-threat s-to-global-health-in-2019

7. McMurtry CM. Managing immunization stress-related response: A contributor to sustaining trust in vaccines. Can Commun Dis Rep 2020;46(6):210-8. DOI PubMed

8. Gold MS, MacDonald NE, McMurtry CM, Balakrishnan MR, Heininger U, Menning L, Benes O, Pless R, Zuber PL. Immunization stress-related response - Redefining immunization anxiety-related reaction as an adverse event following immunization. Vaccine 2020;38(14):3015-20. DOI PubMed 
9. Kikuta A, Gardezi F, Dubey V, Taddio A. Practices and perceptions regarding pain and pain management during routine childhood immunizations: findings from a focus-group study with nurses working at Toronto Public Health, Ontario. Can J Infect Dis Med Microbiol 2011;22(2):43-8. DOI PubMed

10. Gerges S, Peter E, Bowles SK, Diamond S, Bucci LM, Resnick A, Taddio A. Pharmacists as vaccinators: an analysis of their experiences and perceptions of their new role. Hum Vaccin Immunother 2018;14(2):471-7. DOI PubMed

11. Taddio A, McMurtry CM, Bucci LM, MacDonald N, Ilersich AN, Ilersich AL, Alfieri-Maiolo A, deVlaming-Kot C Alderman L; Pain Pain Go Away Team. Overview of a Knowledge Translation (KT) Project to improve the vaccination experience at school: the CARD ${ }^{\mathrm{TM}}$ System. Paediatr Child Health 2019;24 Suppl 1:S3-18. DOI PubMed

12. World Health Organization. Reducing pain at the time of vaccination: WHO position paper - September 2015. Wkly Epidemiol Rec 2015;90(39):505-10. DOI PubMed
13. Freedman $T$, Taddio A, McMurtry CM, Wong $\mathrm{H}$, MacDonald $\mathrm{N}$, McDowall T, deVlaming-Kot C, Alderman L; Pain Pain Go Away Team. Involving stakeholders in informing the development of a Knowledge Translation (KT) intervention to improve the vaccination experience at school. Paediatr Child Health 2019 Apr;24 Suppl 1:S19-28. DOI PubMed

14. Taddio A, Alderman L, Freedman T, McDowall T, McMurtry CM, MacDonald N, deVlaming-Kot C, Alfieri-Maiolo A; Pain Pain Go Away Team. The CARD ${ }^{\text {TM }}$ System for improving the vaccination experience at school: results of a small-scale implementation project on program delivery. Paediatr Child Health 2019 Apr;24 Suppl 1:S54-67. DOI PubMed

15. World Health Organization. WHO best practices for injections and related procedures toolkit. March 2010. Geneva: WHO; 2010. https://apps.who.int/iris/bitstream/han dle/10665/44298/9789241599252_eng.pdf;jsessionid=BCD6 FA7D110B62AB5289824942EC6294?sequence=1

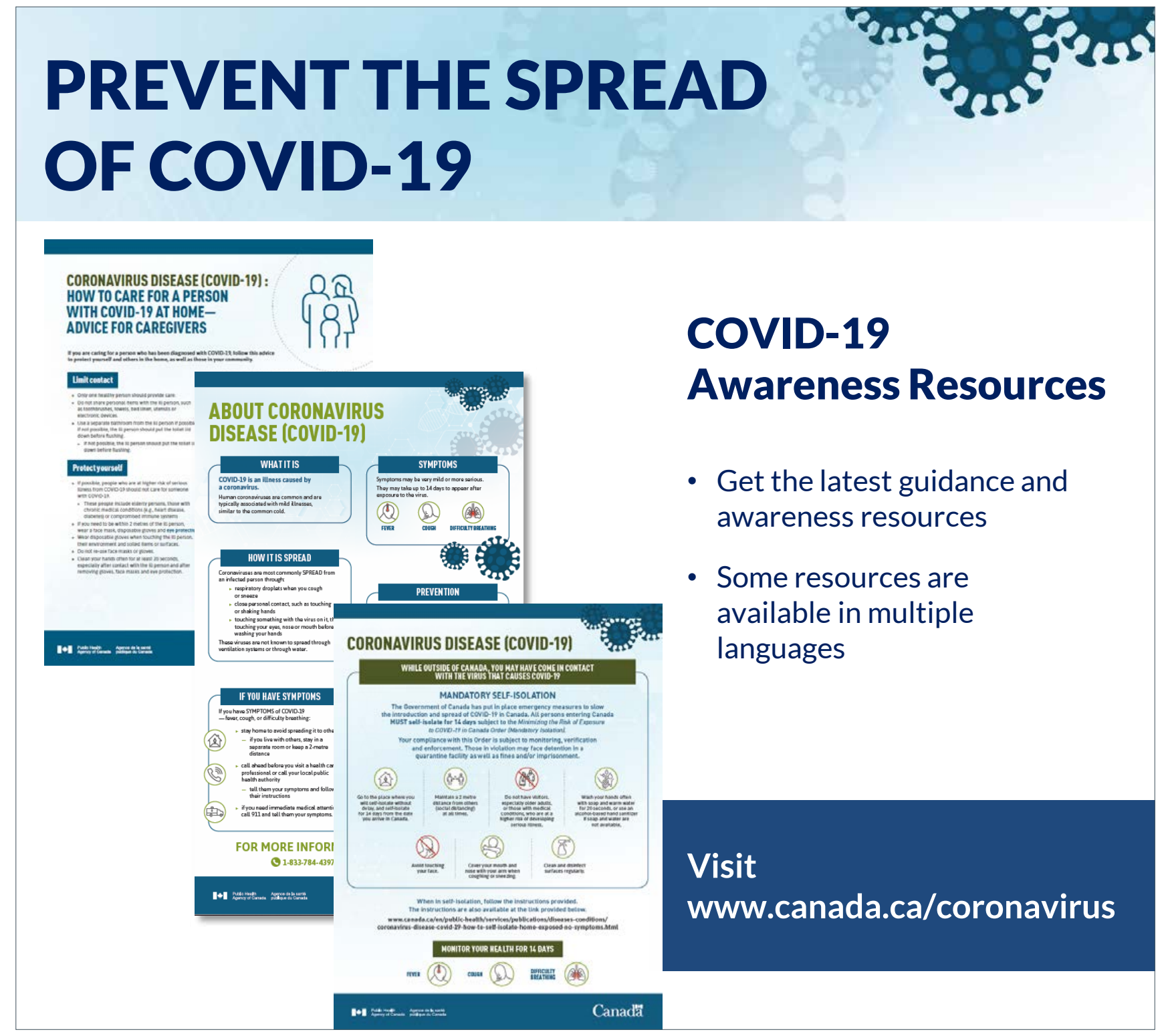

